\title{
Inoculation with the endophyte Piriformospora indica significantly affects mechanisms involved in osmotic stress in rice
}

\author{
Muhammad Abu Bakar Saddique ${ }^{1,3}$, Zulfiqar Ali ${ }^{1,4^{*}}$ D, Abdus Salam Khan ${ }^{1}$, Iqrar Ahmad Rana ${ }^{2}$ \\ and Imran Haider Shamsi ${ }^{3^{*}}$
}

\begin{abstract}
Background: Rice is a drought susceptible crop. A symbiotic association between rice and mycorrhizal fungi could effectively protect the plant against sudden or frequent episodes of drought. Due to its extensive network of hyphae, the endophyte is able to deeply explore the soil and transfer water and minerals to the plant, some of them playing an important role in mitigating the effects of drought stress. Moreover, the endophyte could modify the expression of drought responsive genes and regulate antioxidants.

Results: Three rice genotypes, WC-297 (drought tolerant), Caawa (moderately drought tolerant) and IR-64 (drought susceptible) were inoculated with Piriformospora indica (P. indica), a dynamic endophyte. After 20 days of co-cultivation with the fungus, rice seedlings were subjected to $15 \%$ polyethylene glycol-6000 induced osmotic stress. P. indica improved the growth of rice seedlings. It alleviated the destructive effects of the applied osmotic stress. This symbiotic association increased seedling biomass, the uptake of phosphorus and zinc, which are functional elements for rice growth under drought stress. It boosted the chlorophyll fluorescence, increased the production of proline and improved the total antioxidant capacity in leaves. The association with the endophyte also up regulated the activity of the Pyrroline-5-carboxylate synthase (P5CS), which is critical for the synthesis of proline.
\end{abstract}

Conclusion: A mycorrhizal association between P. indica and rice seedlings provided a multifaceted protection to rice plants under osmotic stress (- $0.295 \mathrm{MPa})$.

Keywords: Oryza sativa, Piriformospora indica, Symbiosis, Osmotic stress, Fv/Fm, Phosphorus, Zinc, Proline, Total antioxidant capacity, Pyrroline-5-carboxylate synthase

\section{Background}

Rice is staple food for nearly half of the world's seven billion people. Almost 754.6 million tons of paddy are annually produced (FAO 2017). Considering the increasing population and the associated demand of rice there is a need to increase this crop's yield. Because of its semiaquatic ancestors, rice is very susceptible to drought stress, which

\footnotetext{
* Correspondence: zulfiqarpbg@hotmail.com; drimran@zju.edu.cn 'Department of Plant Breeding and Genetics, University of Agriculture, 38040, Faisalabad, Pakistan

${ }^{3}$ Department of Agronomy, College of Agriculture and Biotechnology,

Zhejiang University, 310058, Hangzhou, People's Republic of China

Full list of author information is available at the end of the article
}

drastically affects its growth and grain yield (Yue et al. 2006; Fahad et al. 2017). Drought is one of the major cause of yield reduction in rice belts of different countries (FAO 2017). Drought interferes with and damages morphological, physiological and molecular features that are responsible for growth and development (Farooq et al. 2009a). In the case of rice, low water availability reduces germination, plant biomass, number of tillers, plant height and modifies root angle (Ji et al. 2012; Sokoto and Muhammad 2014; Uga et al. 2015). It decreases transpiration, stomatal conductance, water use efficiency, relative water content, chlorophyll content, photosynthesis, photosystem II activity 
and affects membrane stability and abscisic acid content (Ding et al. 2014; Yang et al. 2014). Drought induces the accumulation of osmoprotectants like proline, sugars, polyamines and antioxidants ( $\mathrm{Li}$ et al. 2011; Fahramand et al. 2014) as well as changes in the expression of genes which encode transcription factors and defense related proteins (Nakashima et al. 2014).

Various strategies can help maintaining rice grain yield under drought prone conditions like, exploitation of diversified germplasm (Liu et al. 2015), effective management practices (Haefele et al. 2016) and by exploiting the association of rice with endophytes (Gill et al. 2016). Some progress has been obtained by genetically improving drought tolerance in rice (Serraj et al. 2011). The management of water during rice field preparation and throughout complete crop growth period can help to save water and make it available during drought episodes (Haefele et al. 2016). Moreover, seed priming, foliar application of growth regulators as well as osmoprotectants and the proper application of phosphorus (P), zinc ( $\mathrm{Zn})$ and silicon $(\mathrm{Si})$ can mitigate drought effects under limited water environment (Hattori et al. 2005; Kaya et al. 2006; Taiz and Zeiger 2006; Ashraf and Foolad 2007; Tariq et al. 2017; Gill and Tuteja 2010). Finally, symbiotic association with endophytes can help maintain grain production under stress conditions. Hyphae of the mycorrhizal fungi are able to deeply explore the rhizosphere and transport water and minerals to plant roots and keep roots moist even when there is less water availability (Varma et al. 2013).

Among many endophytes, $P$. indica forms symbiotic association with almost every cultivable crop. It extends its mutualistic link with pteridophytes, bryophytes, angiosperms and gymnosperms (Varma et al. 2001). P. indica was found to have a positive effect on host plants growth under saline environment, water stress, temperature shocks as well as biotic stresses. Under osmotic stresses, $P$. indica increases the cellular osmolarity of plant and maintains turgor (Gill et al. 2016). Along with many drought responsive genes $P$. indica up regulates an important proline synthesizing gene, P5CS (Abo-Doma et al. 2016). Proline is highly soluble and zwitterionic in nature so its higher accumulation do not damage plants. Instead, it stabilizes cellular structures, acts as water substitute through hydrophilic interactions and hydrogen bonding. It also induces drought tolerance by scavenging ROS and by being utilized as energy source after the release of stress (Verslues and Sharma 2010). Under abiotic stress P. indica was also found to stabilize chlorophyll in rice leaves (Abadi and Sepehri 2016). By consequence, it increases the maximum quantum yield of PSII ( $\mathrm{Fv} / \mathrm{Fm})$ (Vahabi et al. 2016; Shahabivand et al. 2017).

Finally, $P$. indica enhances the uptake of $\mathrm{P}$ and $\mathrm{Zn}$. High $\mathrm{P}$ uptake is responsible for maintaining optimum leaf relative water contents, efficiency of photosystem II and net photosynthetic rate. High phosphorus level decreases malondialdehyde content and increases osmolytes and nitrogenous compounds concentration (Tariq et al. 2017). Zinc is part of antioxidant complexes $(\mathrm{Cu} /$ $\mathrm{Zn}$-SOD) and is also important for scavenging ROS that ultimately reduces the damage to cellular membranes (Gill and Tuteja 2010; Ngwene et al. 2016). In response to these valuable services, the plant could devote up to $15-20 \%$ of the produced photosynthates to this fungus but the extent of benefits generally offsets this cost (Varma et al. 2001). In the present study, rice plants have been inoculated with $P$. indica under polyethylene glycol-6000 (PEG-6000) induced water stress in hydroponic conditions. The effects of the symbiotic association under osmotic stress were analyzed on seedling biomass, root and shoot length, $\mathrm{P}$ and $\mathrm{Zn}$ uptake, expression of P5CS gene, integrity of grana in chloroplasts, level of $\mathrm{Fv} / \mathrm{Fm}$, proline content and total antioxidant capacity (TAC). Whereas, in most of the previous research articles, the interaction between rice roots and $P$. indica had been studied under salt and heavy metal stress. It had been reported that this symbiotic fungus enhances the seedling biomass, length of root and shoot, chlorophyll content and proline concentration under salt stress (Jogawat et al. 2013; Bagheri et al. 2013). Previously, the interaction between rice roots and $P$. indica under osmotic stress was not fully explored. We have tried to highlight this interaction and concluded that $P$. indica induces drought tolerance in rice seedlings by improving plant morphological, physiological and molecular aspects.

\section{Methods}

\section{Growth and cultivation conditions}

$P$. indica was grown in Kafer liquid media (Hill and Kafer 2001). The $\mathrm{pH}$ of the media was adjusted at 6.5 and temperature at $28{ }^{\circ} \mathrm{C}$. Growing culture was continuously shaken at $130 \mathrm{rpm}$. Three rice genotypes were used in this study, WC-297 (drought tolerant), Caawa (moderately drought tolerant) and IR-64 (drought susceptible).

The method of Sarma et al. (2011) was used for the effective coating of fungus on rice seeds. Seeds of the genotypes were surface sterilized and coated with a paste of $P$. indica in vermiculite, used as a carrier to adhere spores on seed surface. Seeds without fungus association were treated with only vermiculite. After 10 days of cocultivation with $P$. indica, seedlings were transferred to a Yoshida nutrition media (Yoshida et al. 1976). After 10 days of acclimatization in this media, a 15\% solution of PEG-6000 was added to induce osmotic stress in half of the inoculated and half of the non-inoculated plants. Whereas, 15\% PEG 6000 induces $-0.295 \mathrm{MPa}$ of osmotic pressure at $25{ }^{\circ} \mathrm{C}$ (Michel and Kaufmann 1973). 


\section{Colonization of fungus: Staining of fungal hyphae and spores}

To monitor root colonization, roots were analyzed after 4 days of germination and at the end of the experiment. Roots were thoroughly washed with deionized water, cut into $1 \mathrm{~cm}$ length segments which were placed overnight in a $10 \% \mathrm{KOH}$ solution at room temperature. Roots were then washed 5 times with sterilized $\mathrm{H}_{2} \mathrm{O}$ and incubated in $1 \% \mathrm{HCl}$ solution for $3 \mathrm{~min}$. Later on, roots were mounted in $0.05 \%$ trypan blue in lactophenol for microscopic examination (Michal Johnson et al. 2011).

\section{Morphological analysis}

Root and leaf samples were collected 20 days after the beginning of stress exposure. Under control and osmotic stress the gain in fresh root weight (FRW), fresh shoot weight (FSW), dry root weight (DRW), dry shoot weight (DRW), root length (RL) and shoot length (SL) was determined both from non-inoculated and inoculated plants.

\section{Elemental analysis of leaves and roots}

Root and leaf samples of the rice plants were collected 20 days after the beginning of stress exposure. Samples were dried in a hot air oven, initially for $3 \mathrm{~h}$ at $105^{\circ} \mathrm{C}$ and then for another $24 \mathrm{~h}$ at $80{ }^{\circ} \mathrm{C}$. Approximately $0.1 \mathrm{~g}$ of dried leaves and roots was used to determine the concentration of $\mathrm{P}$ and $\mathrm{Zn}$. Samples were digested in $5 \mathrm{ml}$ of $\mathrm{HNO}_{3}$ at $120{ }^{\circ} \mathrm{C}$ for $2 \mathrm{~h}$ and then at $80{ }^{\circ} \mathrm{C}$ for another $2 \mathrm{~h}$ in microwave digester (Microwave 3000; Antoon Paar). After digestion, they were diluted to $20 \mathrm{ml}$ with Mili-Q water, and the elemental contents were determined using an inductively coupled plasma-optical emission spectrometer (ICP-OES;. Optima 8000DV; PerkinElmer).

\section{Determining the chlorophyll fluorescence}

Drought induced change in Fv/Fm was determined 20 days after the beginning of stress exposure, using a fluorometer (IMAG-MAXI, Heinz Walz, Effeltrich, Germany). Plants were placed in the dark for $30 \mathrm{~min}$, then $20 \mathrm{~cm}$ long leaves were cut and immediately analyzed.

\section{Electron microscopy observation of chloroplasts}

Chloroplasts in rice leaves were examined 20 days after the beginning of stress exposure, using a transmission electron microscope (Hitachi Model H-7650 TEM). Samples were first fixed in $2.5 \%$ glutaraldehyde in phosphate buffer $(0.1 \mathrm{M}, \mathrm{pH}$ 7.0) for more than $4 \mathrm{~h}$ and washed three times with phosphate buffer $(0.1 \mathrm{M}, \mathrm{pH} 7$. 0 ) for $15 \mathrm{~min}$. They were fixed with $1 \% \mathrm{OsO}_{4}$ in phosphate buffer $(0.1 \mathrm{M}, \mathrm{pH} 7.0)$ for $2 \mathrm{~h}$, and washed three times in phosphate buffer (0.1 M, pH 7.0) for $15 \mathrm{~min}$. They were then dehydrated stepwise using graded ethanol (30\%, 50\%, 70\%, 80\%, 90\%, 95\% and 100\%) for $15 \mathrm{~min}$ at each step and transferred to absolute acetone for $20 \mathrm{~min}$. Infiltration of the samples was done by placing them in a mixture of absolute acetone and the final spurr resin mixture $(1: 1)$ for $1 \mathrm{~h}$ at room temperature. Then samples were transferred to a mixture of absolute acetone and final resin mixture (1:3) for $3 \mathrm{~h}$ and to final spurr resin mixture for overnight. The samples were finally placed in eppendorf tubes containing spurr resin and heated at $70{ }^{\circ} \mathrm{C}$ for more than $9 \mathrm{~h}$. They were sectioned with a LEICA EM UC7 ultratome and sections were stained with uranyl acetate and alkaline lead citrate for 5 and $10 \mathrm{~min}$ respectively and then observed in transmission electron microscope (Hitachi Model $\mathrm{H}$ 7650).

\section{Expression pattern of P5CS gene}

The expression pattern of P5CS (proline synthesizing gene) was observed $24 \mathrm{~h}$ after the beginning of stress exposure. For primers designing, coding DNA sequences (CDS) were retrieved from The Rice Annotation Project Database (http://rapdb.dna.affrc.go.jp/). Ubiquitin $(U B Q)$ was used as a reference gene. Forward and reverse primers of these two genes are given in the Additional file 1: Table S1. TRIzol $^{\oplus}$ reagent (FAVORGEN BIOTECH CORP) was used for the extraction of RNA. Plant leaves were grind in liquid nitrogen and then transferred immediately in microfuge tube holding $700 \mu \mathrm{l}$ Trizol. This mixture was centrifuged at $12000 \mathrm{rpm}$ for $2 \mathrm{~min}$. Supernatant was shifted in another tube and $300 \mu \mathrm{l}$ chloroform was added and mixed gently by inversion. The solution was centrifuged to get phase separation at $12000 \mathrm{rpm}$ for $10 \mathrm{~min}$. The upper phase was collected in another microfuge tube, an equal amount of iso-propanol was added followed by an incubation on ice for $10 \mathrm{~min}$. Again centrifuged at $12000 \mathrm{rpm}$ for $10 \mathrm{~min}$, supernatant was discarded and pellet of RNA was collected. Pellet was washed with 75\% ethanol. Air dried for 2-3 min and diluted in $25 \mu \mathrm{lnu}-$ clease free water. All centrifugations were done at $4{ }^{\circ} \mathrm{C}$. High quality RNA was used to synthesize cDNA following the protocol of Thermo Fisher Scientific. Gel bands of the PCR products were analyzed with IMAGE. J software. Numerical values showing gel band strength were used to calculate the percentage of change in the expression of P5CS.

\section{Proline analysis}

For the determination of proline, the method of Bates et al. (1973) was used. Leaves (0.5 g) collected 20 days after the beginning of stress exposure, were homogenized in $5 \mathrm{~mL}$ of $3 \%$ aqueous sulphosalicylic acid. The homogenate was filtered through Whatman No.2 filter paper. One milliliter of filtrate was taken and mixed with $1 \mathrm{~mL}$ of acid ninhydrin and $1 \mathrm{~mL}$ of glacial acetic acid in a test tube. The mixture was briefly vortexed and heated at 
$100{ }^{\circ} \mathrm{C}$ in a water bath for $1 \mathrm{~h}$ and terminated the reaction in an ice bath. Four milliliter of toluene were added to the solution and vortexed for $15-20 \mathrm{~s}$. The chromophore containing free proline was aspirated from the aqueous phase in a test tube and warmed to room temperature. The absorbance was measured at $520 \mathrm{~nm}$ using a spectrophotometer.

\section{Estimation of total antioxidant capacity}

The method of Prieto et al. (1999) was used for the determination of total antioxidant capacity (TAC). Leaf samples $(0.4 \mathrm{~g})$ collected 20 days after the beginning of stress exposure, were homogenized in 50\% methanol. The assay mixture containing $900 \mu$ l reagent $(0.6 \mathrm{M}$ $\mathrm{H}_{2} \mathrm{SO}_{4}, 28 \mathrm{mM}$ sodium phosphate and $4 \mathrm{mM}$ ammonium molybdate) and $100 \mu \mathrm{l}$ of enzyme extract was boiled at $95{ }^{\circ} \mathrm{C}$ for $30 \mathrm{~min}$. Spectrophotometer absorbance was measured at $695 \mathrm{~nm}$, a higher absorbance (nm) indicating a higher TAC (Prasad et al. 2009).

\section{Statistical analysis}

Experiment was performed by following factorial under completely randomized design. It had three replications (each replication had one plant per pot), three genotypes and four treatments (no osmotic stress, no osmotic stress $+P$. indica inoculation, osmotic stress and osmotic stress $+P$. indica inoculation). Data were analyzed using Analysis of Variance method and least significant difference $\left(\mathrm{LSD}_{0.05}\right)$ (Statistix 8 , version 8.1).

\section{Results}

\section{Establishment of symbiotic association}

$P$. indica successfully colonized rice roots. A strong association of root and fungus was observed after 4 days of germination. Fungus was found to develop spores and hyphae within emerging roots (Fig. 1a). This association was maintained until the end of experiment (Fig. 1b).

\section{Morphological analysis}

Seedling biomass and length of root and shoot were significantly improved in inoculated plants $(p \leq 0$. 01, Table 1). In the absence of osmotic stress, the average FRW of the non-inoculated plants of WC-297, Caawa and IR-64 was 1.73, 1.82 and 1.72 g, respectively. When plants were inoculated with $P$. indica the average FRW was increased to $1.86,2.06$ and $2.24 \mathrm{~g}$ in the three genotypes, respectively. Under osmotic stress average FRW in WC-297, Caawa and IR-64 was 0.75, 0.61 and 0. $59 \mathrm{~g}$ in non-inoculated plants and $1.02,0.82$ and $0.94 \mathrm{~g}$ in inoculated plants (Fig. 2a). In the absence of osmotic stress, the average FSW of non-inoculated plants were 3 . 44, 3.38 and $3.01 \mathrm{~g}$ in WC-297, Caawa and IR-64, respectively. In inoculated plants it was $3.79,3.82$ and 3 . $49 \mathrm{~g}$ in WC-297, Caawa and IR-64, respectively. Under osmotic stress the average FSW for the non-inoculated plants of these three genotypes was $1.61,1.20$ and $1.09 \mathrm{~g}$ and in inoculated plants it was $1.82,1.37$ and $1.53 \mathrm{~g}$ in WC-297, Caawa and IR-64, respectively (Fig. 2b). In the absence of osmotic stress, the average DRW of the noninoculated plants of WC-297, Caawa and IR-64 was 0. $21,0.26$ and $0.22 \mathrm{~g}$, respectively. When plants were inoculated with $P$. indica the average DRW was increased to $0.28,0.29$ and $0.31 \mathrm{~g}$ in the three genotypes, respectively. Under osmotic stress average DRW in WC-297, Caawa and IR-64 was $0.12,0.09$ and $0.11 \mathrm{~g}$ in non-inoculated plants and $0.18,0.10$ and $0.15 \mathrm{~g}$ in inoculated plants, respectively (Fig. 2c). In the absence of osmotic stress, the average DSW of non-inoculated plants were $0.49,0.52$ and $0.49 \mathrm{~g}$ in WC-297, Caawa and IR-64, respectively. In inoculated plants it was $0.64,0.53$ and $0.49 \mathrm{~g}$ in WC297, Caawa and IR-64, respectively. Under osmotic stress
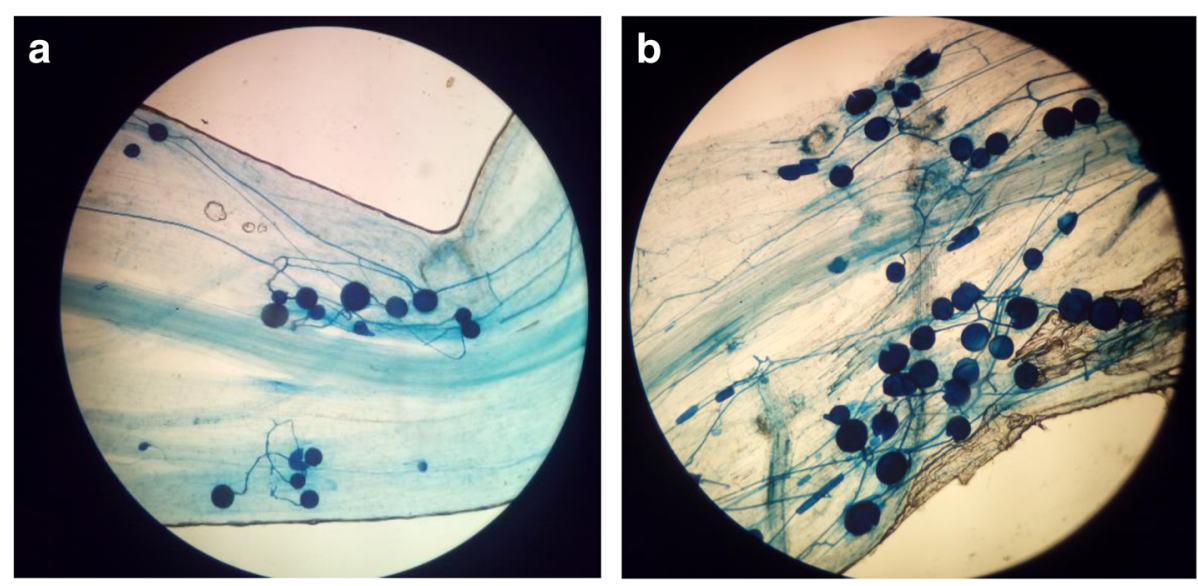

Fig. 1 Association of $P$. indica with rice roots. a Established symbiosis after 4 days of germination, a network of hyphae and spores is visible within rice roots. $\mathbf{b}$ Network of hyphae along with attached spores, observed at the end of experiment 
Table 1 Mean squares of absolute values for various seedling traits of the three rice genotypes grown in control, control $+P$. indica, drought and drought $+P$. indica

\begin{tabular}{lllll}
\hline Residual & Genotype $\times$ Treatment & Treatment & Genotype & Source of variation \\
\hline 24 & 6 & 3 & 2 & df \\
0.0018 & $0.055^{* *}$ & $3.9^{* *}$ & $0.0066^{* *}$ & Fresh root weight \\
0.0035 & $0.067^{* *}$ & $13^{* *}$ & $0.45^{*}$ & Fresh shoot weight \\
0.000024 & $0.0027^{* *}$ & $0.068^{* *}$ & $0.00051^{* *}$ & Dry root weight \\
0.00011 & $0.0076^{* *}$ & $0.21^{* *}$ & $0.0035^{* *}$ & Dry shoot weight \\
1.7 & $17^{* *}$ & $306^{* *}$ & $61^{* *}$ & Root length \\
3.7 & $14^{*}$ & $202^{* *}$ & $7.2^{\mathrm{NS}}$ & Shoot length \\
0.0000073 & $0.00066^{* *}$ & $0.0050^{* *}$ & $0.0070^{* *}$ & Fv/Fm \\
5.7 & $229^{* *}$ & $1333^{* *}$ & $1088^{* *}$ & Proline \\
0.00045 & $0.0056^{* *}$ & $0.042^{* *}$ & $0.0085^{* *}$ & Total antioxidant capacity \\
0.0086 & $0.68^{* *}$ & $22^{* *}$ & $0.015^{\mathrm{NS}}$ & Phosphorus in root \\
0.018 & $0.056^{*}$ & $20^{* *}$ & $0.14^{* *}$ & Phosphorus in shoot \\
0.0000012 & $0.000058^{* *}$ & $0.00046^{* *}$ & $0.000034^{* *}$ & Zinc in root \\
0.0000029 & $0.00018^{* *}$ & $0.0020^{* *}$ & $0.0000087^{\mathrm{NS}}$ & Zinc in shoot \\
\hline
\end{tabular}

*** and NS indicates significant differences at $p \leq 0.05, p \leq 0.01$ and non-significant $(p>0.05)$, respectively

the DSW for the non-inoculated plants of these three genotypes was $0.21,0.25$ and $0.21 \mathrm{~g}$ and in inoculated plants it was 0.33, 0.27 and $0.35 \mathrm{~g}$ in WC-297, Caawa and IR-64, respectively (Fig. 2d). In the absence of osmotic stress, the average RL of the non-inoculated plants of WC-297, Caawa and IR-64 was 39.33, 36.33 and 35. $34 \mathrm{~cm}$, respectively. When plants were inoculated with $P$. indica the average RL was increased to 46.0, 45.0 and $43.0 \mathrm{~cm}$ in the three genotypes, respectively. Under osmotic stress average RL in WC-297, Caawa and IR-64 was $44.6,30.7$ and $34.6 \mathrm{~cm}$ in non-inoculated plants and $48.0,47.6$ and $46.0 \mathrm{~cm}$ in inoculated plants, respectively (Fig. 2e). In the absence of osmotic stress, the average SL of non-inoculated plants were 37.0, 34.6 and $37.7 \mathrm{~cm}$ in WC-297, Caawa and IR-64, respectively. In inoculated plants it was $41.3,42.3$ and $41.0 \mathrm{~cm}$ in WC-297, Caawa and IR-64, respectively. Under osmotic stress the average SL for the non-inoculated plants of these three genotypes was $37.6,31.0$ and $33.0 \mathrm{~cm}$ and in inoculated plants it was $43.6,45.0$ and $43.0 \mathrm{~cm}$ in WC-297, Caawa and IR-64, respectively (Fig. 2f).

\section{Elemental analysis}

The concentration of $\mathrm{P}$ and $\mathrm{Zn}$ were significantly improved in the presence of root symbiosis $(p \leq 0.01$, Table 1$)$. The uptake of $\mathrm{P}$ was almost double in inoculated than in noninoculated plants. In the absence of osmotic stress, average $\mathrm{P}$ concentration in leaves of the non-inoculated plants of WC-297, Caawa and IR-64 was 1.85, 1.88 and 1.95 mg.g ${ }^{-1}$, respectively. When plants were inoculated with $P$. indica, $\mathrm{P}$ concentration increased to $4.86,4.38$ and $4.84 \mathrm{mg}^{-g^{-1}}$ in the three genotypes, respectively. Under osmotic stress, $\mathrm{P}$ leaf concentration in WC-297, Caawa and IR-64 was 1.3, 1.
13 and 1.16 mg.g ${ }^{-1}$ in non-inoculated plants, and 2.19, 2.16 and $2.42 \mathrm{mg}^{-1} \mathrm{~g}^{-1}$ in inoculated plants (Fig. 3a). Almost similar change in $\mathrm{P}$ level was noticed when roots were analyzed. In the absence of osmotic stress, P concentration in roots of non-inoculated plants were $1.71,1.65$ and 1. $81 \mathrm{mg} \cdot \mathrm{g}^{-1}$ in WC-297, Caawa and IR-64, respectively. In inoculated plants, $\mathrm{P}$ concentration increased to $4.48,5.51$ and 4.56 mg.g ${ }^{-1}$ in WC-297, Caawa and IR-64, respectively. Under osmotic stress $\mathrm{P}$ concentration of the three genotypes was $1.26,1.14$ and $1.49 \mathrm{mg}^{-g^{-1}}$ in non-inoculated plants and 2.90, 1.76 and $2.35 \mathrm{mg} \cdot \mathrm{g}^{-1}$ in inoculated plants in WC-297, Caawa and IR-64, respectively (Fig. 3b).

The symbiotic association of the rice plant with $P$. indica also increased leaves and roots $\mathrm{Zn}$ concentration. In the absence of osmotic stress, leaves $\mathrm{Zn}$ concentration of non-inoculated plants of WC-297, Caawa and IR-64 was $0.042,0.041$ and $0.048 \mathrm{mg}^{-1} \mathrm{~g}^{-1}$ respectively. In inoculated plants, $\mathrm{Zn}$ concentration increased to 0.068 , 0.084 and $0.067 \mathrm{mg} \cdot \mathrm{g}^{-1}$ in the three genotypes, respectively. Under osmotic stress, the average leaf $\mathrm{Zn}$ concentration of non-inoculated plants of WC-297, Caawa and IR-64 were 0.040, 0.034 and 0.041 mg.g ${ }^{-1}$, respectively. In inoculated plants, leaf $\mathrm{Zn}$ concentration increased to $0.06,0.045$ and $0.055 \mathrm{mg} \cdot \mathrm{g}^{-1}$ in the three genotypes, respectively (Fig. 3c). In the absence of osmotic stress, root $\mathrm{Zn}$ concentration was $0.043,0.045$ and $0.051 \mathrm{mg} . \mathrm{g}^{-1}$, in non-inoculated plants and 0.061, 0 . 05 and $0.054 \mathrm{mg}^{-1} \mathrm{~g}^{-1}$ in inoculated plants of WC-297, Caawa and IR-64, respectively. Under osmotic stress, root $\mathrm{Zn}$ concentration was $0.039,0.034$ and $0.039 \mathrm{mg} . \mathrm{g}^{-}$

1 in non-inoculated plants and $0.042,0.047$ and 0 . $044 \mathrm{mg} \cdot \mathrm{g}^{-1}$ in inoculated plants of WC-297, Caawa and IR-64, respectively (Fig. 3d). 


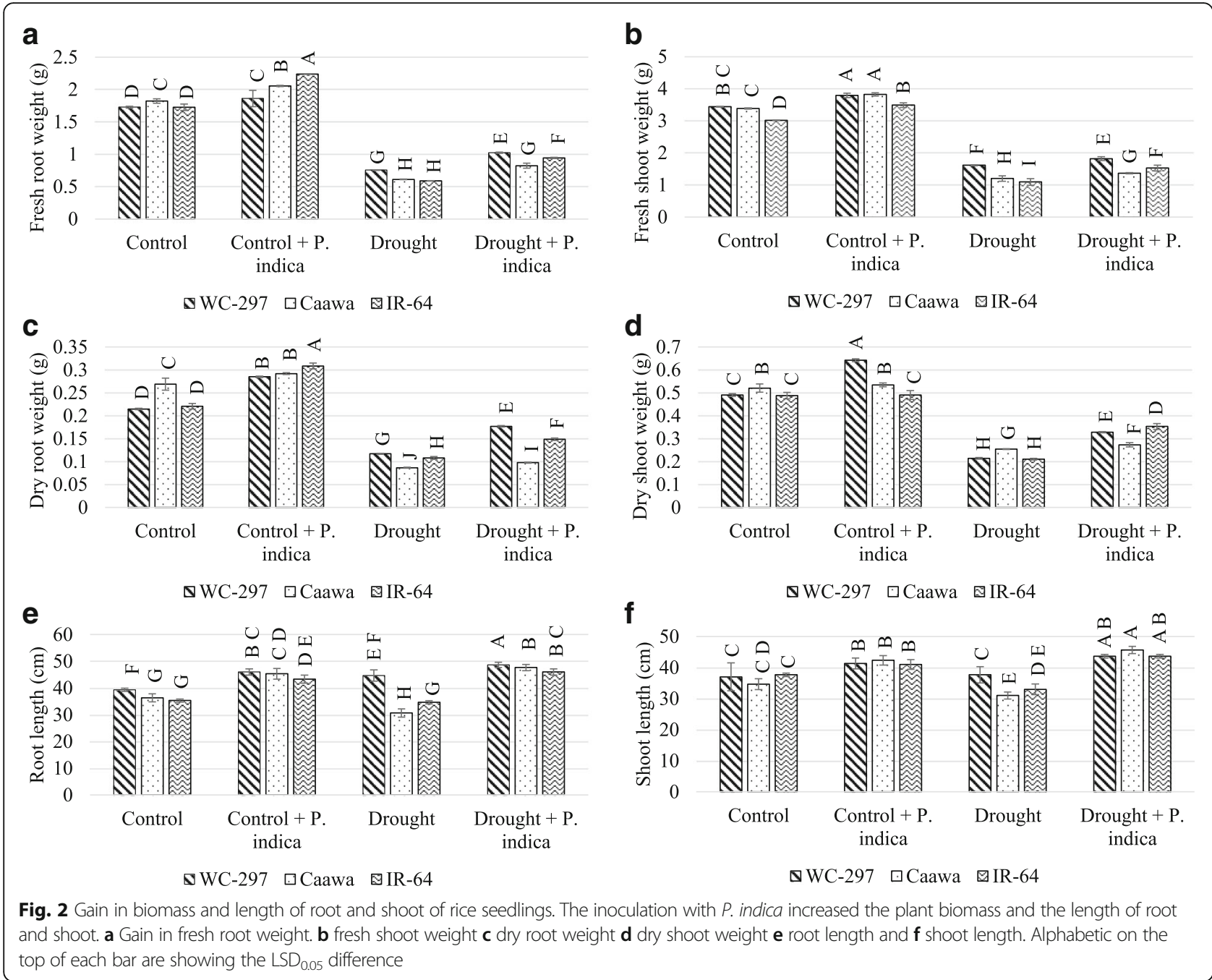

\section{Expression pattern of $P 5 C S$ gene}

The expression of P5CS gene was influenced by the presence of $P$. indica. The change in the expression of $P 5 C S$ was obvious both in the presence and absence of osmotic stress (Fig. 4). In the absence of osmotic stress, the inoculation by $P$. indica increased the expression of this gene up to 102 and 90\% in WC-297 and Caawa, respectively. In IR-64 a decrease of $1.5 \%$ was noted in the expression of P5CS. Under osmotic stress, symbiotic association also increased the expression of P5CS gene by 11, 46 and 64\% in WC-297, Caawa and IR-64, respectively. Osmotic stress induced an increase of the expression of P5CS in non-inoculated plants of WC-297 and Caawa (41\% and 36\%, respectively) and decrease of this expression (43\%) in IR-64.

\section{Proline concentration and total antioxidant capacity}

The concentration of proline and the level of TAC were significantly improved in the presence of root symbiosis $(p \leq 0.01$, Table 1). Osmotic stress increased the level of both proline and TAC in rice leaves, while the inoculation of the plant with $P$. indica boosted the concentration of these beneficial compounds. In the absence of osmotic stress, proline concentration in noninoculated plants was 19.96, 17.92 and $18.83 \mathrm{ppm}$ in WC-297, Caawa and IR-64, respectively. In inoculated plants, proline concentration was increased to 33.94, 23.94 and $25.18 \mathrm{ppm}$. Under osmotic stress proline concentration was $41.31,21.74$ and $20.23 \mathrm{ppm}$ in noninoculated plants and 70.23, 42.81 and $29.96 \mathrm{ppm}$ in inoculated plants, in WC-297, Caawa and IR-64, respectively (Fig. 5a). Plant analysis for TAC also shown similar results. In the absence of osmotic stress, TAC in non-inoculated plants was $0.34,0.29$ and $0.35 \mathrm{~nm}$ in WC-297, Caawa and IR-64, respectively. In inoculated plants TAC was $0.49,0.43$ and $0.36 \mathrm{~nm}$ in WC-297, Caawa and IR-64, respectively. In the presence of osmotic stress TAC was $0.33,0.31$ and $0.37 \mathrm{~nm}$ in noninoculated plants and $0.51,0.46$ and $0.42 \mathrm{~nm}$ in inoculated plants, in WC-297, Caawa and IR-64, respectively (Fig. 5b). 


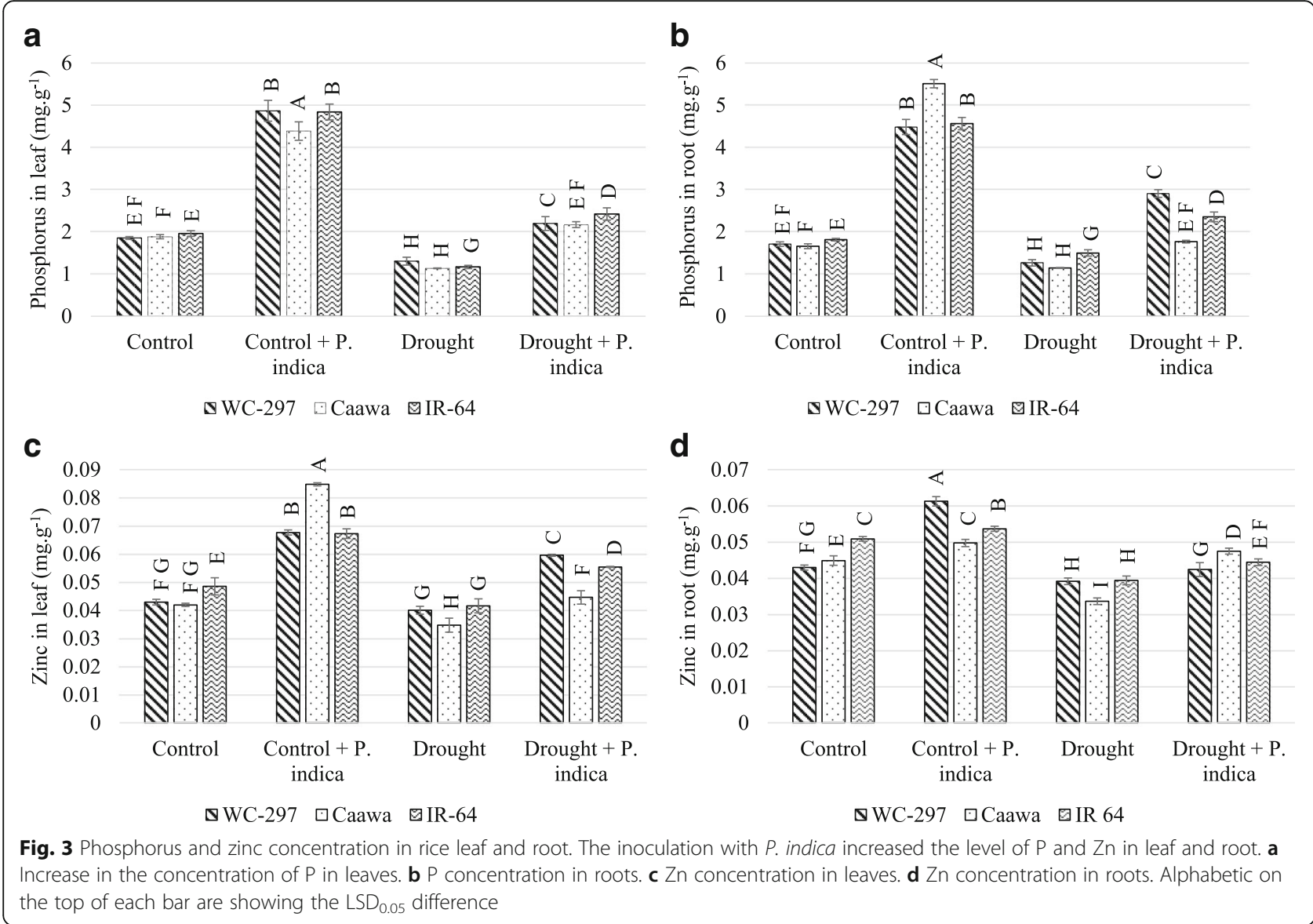

\section{Chlorophyll fluorescence (Fv/Fm)}

$\mathrm{Fv} / \mathrm{Fm}$ was significantly improved in the presence of root symbiosis $(p \leq 0.01$, Table 1$)$. Water limited environment affected the photosynthetic machinery and so, reduced $\mathrm{Fv} / \mathrm{Fm}$. This decrease was higher in IR-64 than in Caawa and WC-297. The symbiotic association increased $\mathrm{Fv} / \mathrm{Fm}$ both in the presence and absence of osmotic stress. In the absence of osmotic stress, the value of $\mathrm{Fv} / \mathrm{Fm}$ in noninoculated plants of WC-297, Caawa and IR-64 was 0.79, 0.81 and 0.81 , respectively. In inoculated plants, it was 0 . $82,0.82$ and 0.81 in the three genotypes. In the presence of osmotic stress, Fv/Fm was $0.79,0.75$ and 0.74 in noninoculated plants and 0.80, 0.80 and 0.78 in inoculated plants, in WC-297, Caawa and IR-64, respectively (Fig. 6).

\section{Electron microscopy}

The inoculation with $P$. indica also stabilized the stacking of grana in chloroplasts in both the absence and presence of osmotic stress. In the absence of osmotic stress the grana were more organized when plants were inoculated with $P$. indica (Fig. 7). Similarly, under osmotic stress the grans were healthy when plants were inoculated with $P$. indica (Fig. 8). In non-inoculated plants, under osmotic stress, the highest damage to chloroplasts was noted in IR-64. Some damage was also observed in Caawa. In WC-297 the chloroplasts were healthy even under drought stress. In inoculated plants, the grana in chloroplast were well organized under osmotic stress.

\section{Discussion}

The effects of osmotic stress have been frequently reported in rice. Osmotic stress hinders mineral uptake, transport and distribution (Zain et al. 2014), reduces chlorophyll content, disintegrate the grana in chloroplasts and disturbs photosynthetic efficiency (Farooq et al. 2009b; Zain et al. 2014; Swapna and Shylaraj 2017; Korres et al. 2017). Several research papers has been published during the last two decades which emphasize the supportive role of $P$. indica in mitigating the osmotic stress in different host plants. It has been reported that $P$. indica serves as a bioregulator, biofertilizer and bioprotector for many host plants. Regardless of the stress, $P$. indica has evolved to protect its food source-the plant. It activates different signaling, transport, metabolic and developmental programs in plants (Ngwene et al. 2016; Gill et al. 2016; Bakshi et al. 2017). We demonstrate that $P$. indica increased the rice seedling biomass, 


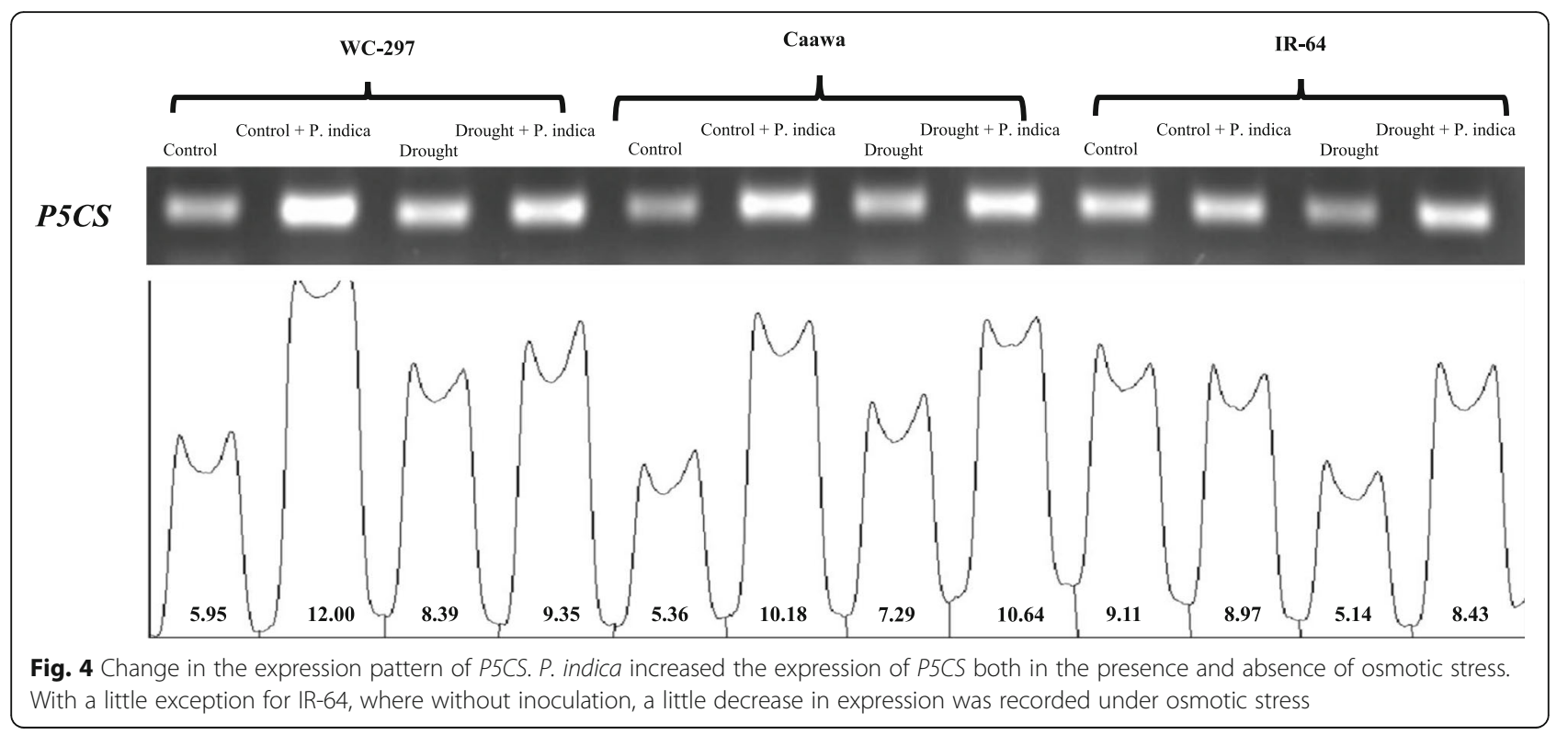

length of root and shoot, $\mathrm{P}$ and $\mathrm{Zn}$ concentration in root and shoot, proline concentration, $\mathrm{TAC}, \mathrm{Fv} / \mathrm{Fm}$ and the expression of P5CS. Most of the previous research articles elaborated the mechanisms that how $P$. indica ameliorates the drastic effects of salt, heavy metal and some biotic stresses in rice plant. It had been reported that this symbiotic fungus increase the concentration of proline, antioxidants and decreases the concentration of malondialdehyde under these stresses (Bagheri et al. 2014; Nassimi and Taheri 2017; Mohd et al. 2017). Most of the yield damage in rice is due to the water shortage. There is very less literature available explaining the rice and $P$. indica symbiosis under osmotic stress. So, there is a need to investigate the role of $P$. indica for inducing drought tolerance in rice. This research article highlighted the $P$. indica induced improvement in some of the important features of rice plants under osmotic stress. We determined that $P$. indica significantly increased the rice plant biomass and the length of root and shoot under control and osmotic stress. Similar growth improvement was reported in Arabidopsis and many other field crops (Franken 2012; Im et al. 2002). In our study, the analysis of mineral profile showed that symbiotic association almost doubled the concentration of both $\mathrm{P}$ and $\mathrm{Zn}$ in leaves and roots of the rice plant. This increase in mineral acquisition was observed in all three genotypes both in the presence and absence of osmotic stress. The increase in the availability of both macro and micronutrients in the rhizosphere due to the association with $P$. indica has been reported in many crops (Franken 2012). Present study, focused on the uptake of $\mathrm{P}$ and $\mathrm{Zn}$ as these two elements have been

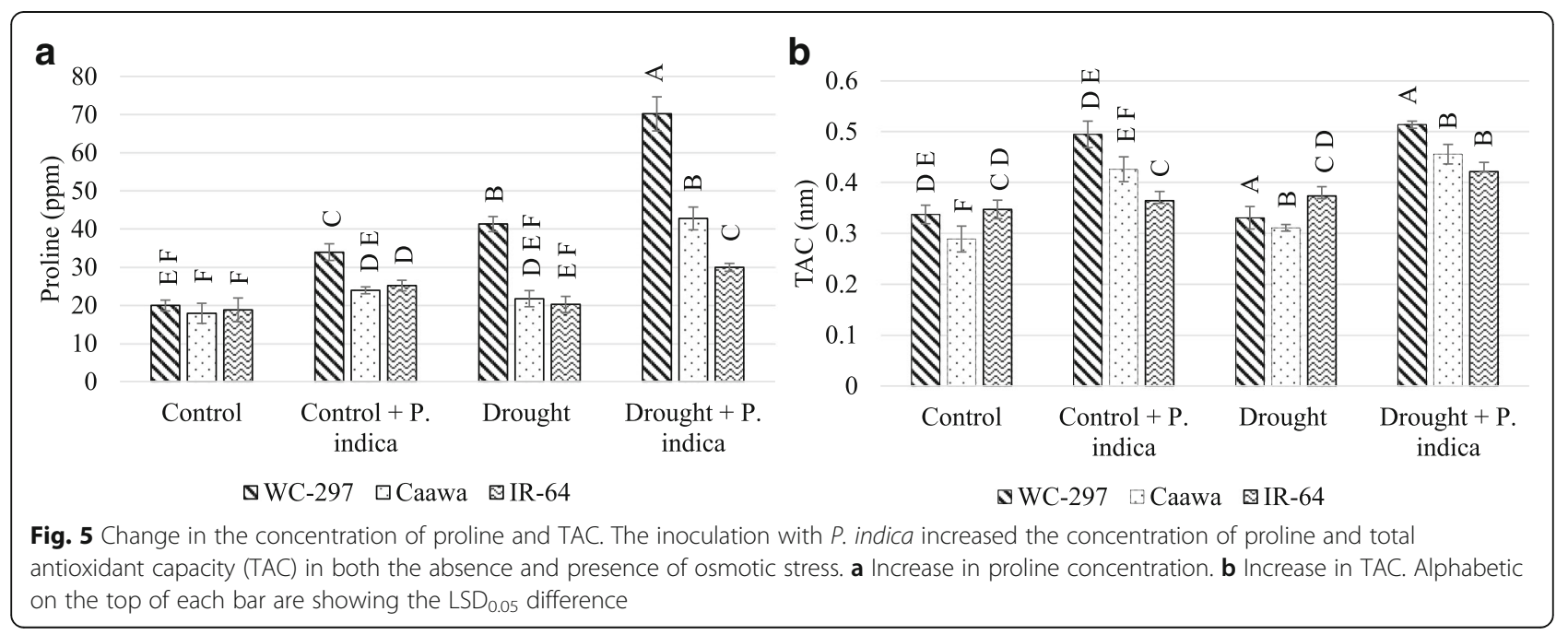




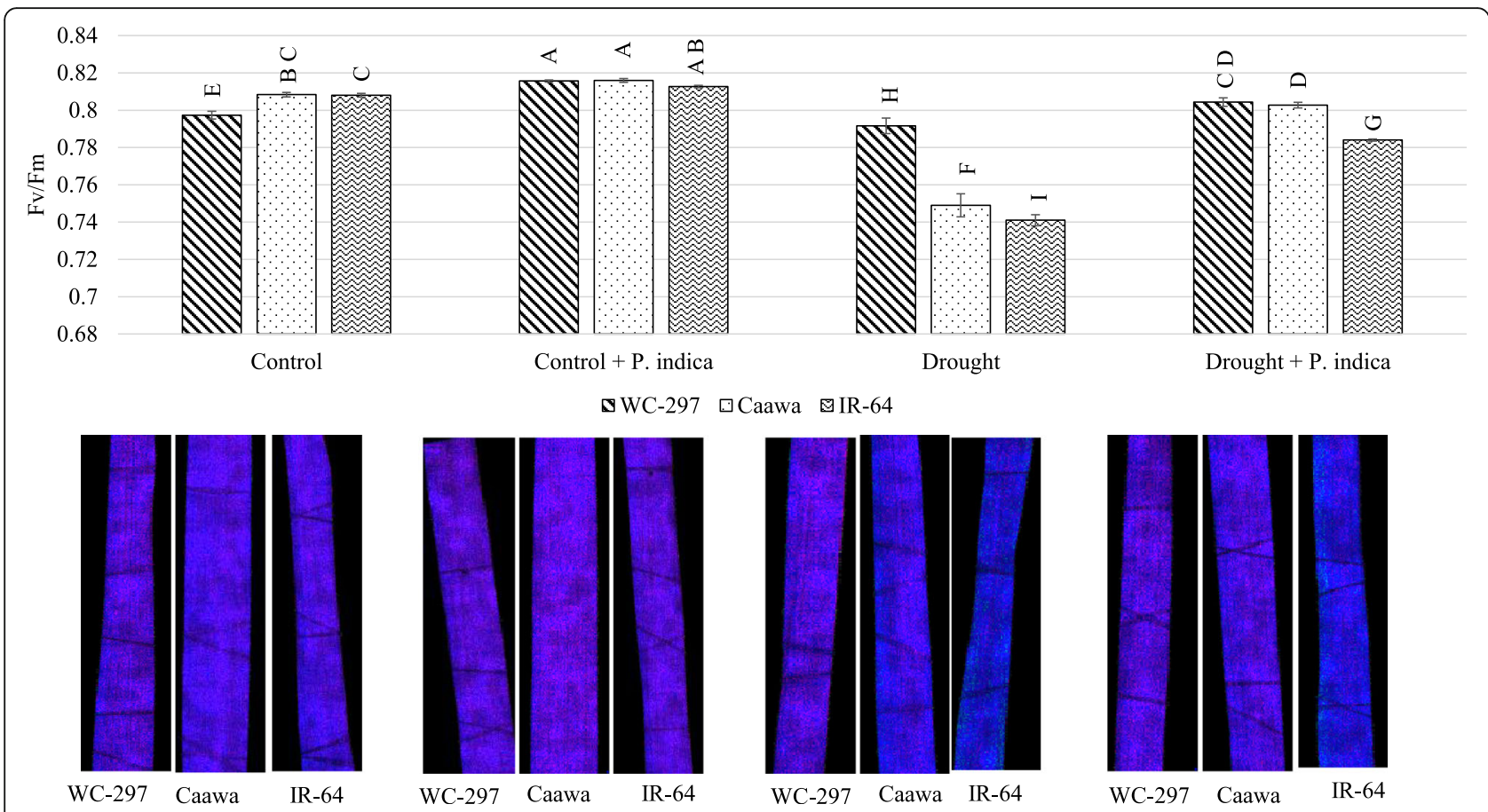

Fig. 6 Change in Fv/Fm. The inoculation with $P$. indica increased Fv/Fm in both the absence and presence of osmotic stress. Under each bar of the graph there is a picture of the leaf section showing Fv/Fm activity. Alphabetic on the top of each bar are showing the $\mathrm{LSD}_{0.05}$ difference

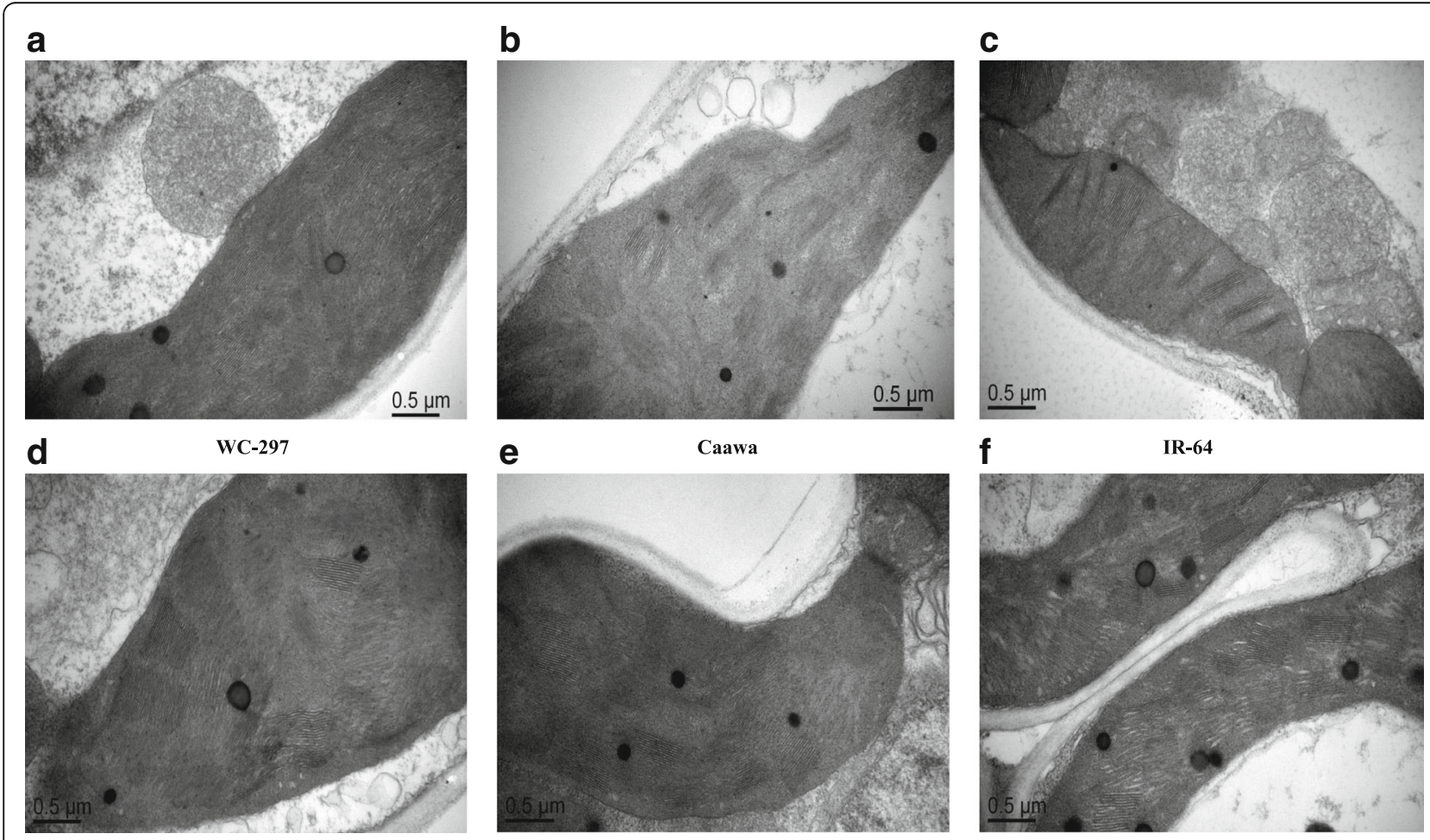

Fig. 7 Chloroplast of rice genotypes in the absence of osmotic stress. a-c Chloroplasts of the non-inoculated plants of WC-297, Caawa and IR-64, respectively. $\mathbf{d}$-f Chloroplasts of the inoculated plants of WC-297, Caawa and IR-64, respectively. Grana in chloroplasts are better organized in rice plants inoculated with $P$. indica 

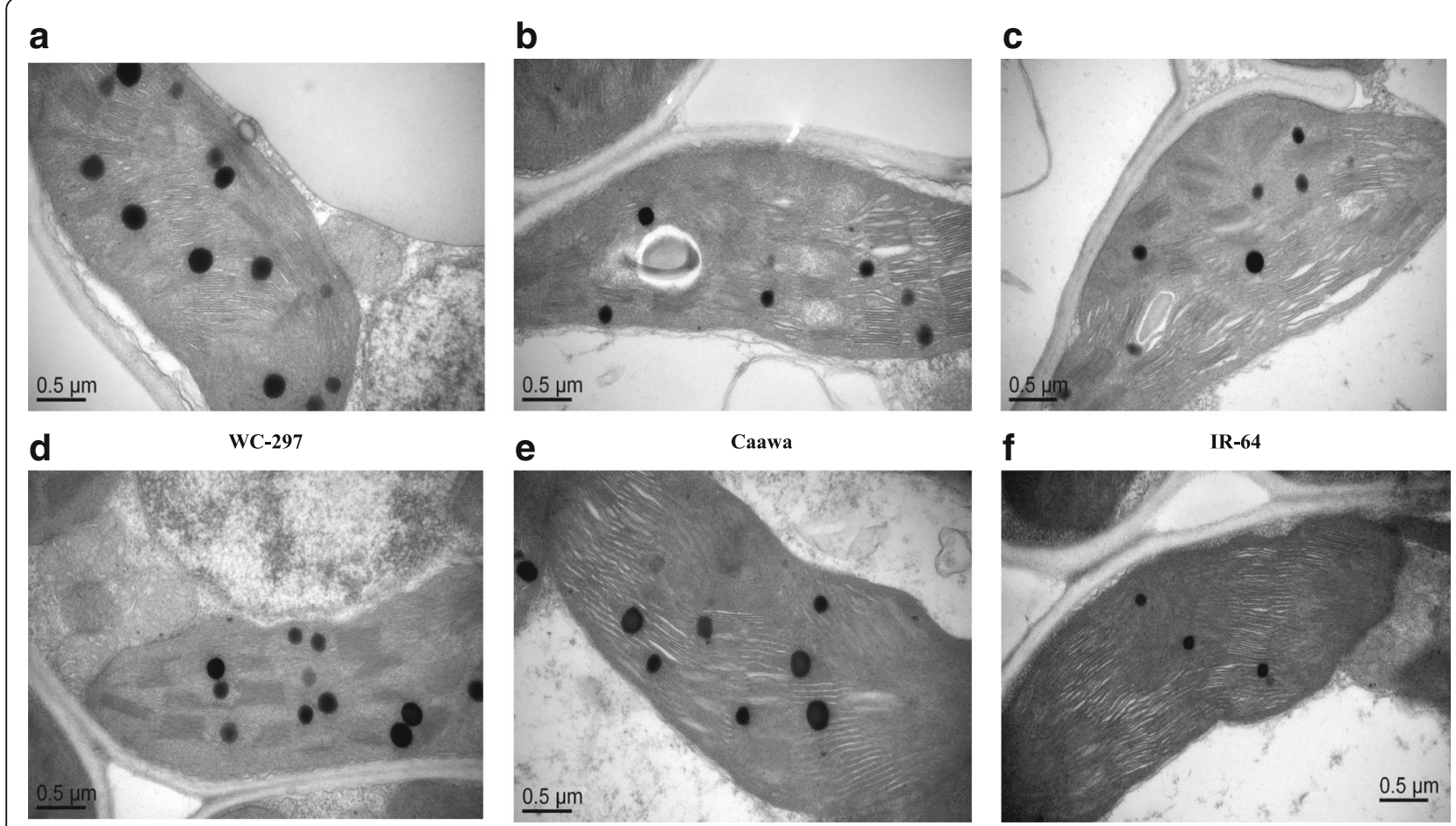

Fig. 8 Chloroplast of rice genotypes in the presence of osmotic stress. a-c Chloroplasts of the non-inoculated plants of WC-297, Caawa and IR-64, respectively. $\mathbf{d}$-f Chloroplasts of the inoculated plants of WC-297, Caawa and IR-64, respectively. Grana in chloroplasts are better organized in rice plants inoculated with $P$. indica

described to have an important role in maintaining plant growth and development under water-limited environments (Ngwene et al. 2016; Tariq et al. 2017). Sufficient supply of $\mathrm{P}$ increases drought tolerance by inducing deeper rooting and higher inorganic phosphorus (Pi) supply for carbon assimilation in leaves. It is also responsible for maintaining optimum leaf relative water contents (Tariq et al. 2017). Similarly, $\mathrm{Zn}$ is critical for the growth of rice plants under stress environments as it is the part of antioxidative enzymes like SOD and CAT. Proper concentration of $\mathrm{Zn}$ in soil helps plant avoiding $\mathrm{ROS}$ damage under abiotic stresses (Cakmak 2000). The effect of the inoculation with $P$. indica on $\mathrm{P}$ and $\mathrm{Zn}$ concentration, observed in this study, has also been reported in other crops by Franken (2012) and Padash et al. (2016).

$\mathrm{Fv} / \mathrm{Fm}$ is an important marker to determine the effect of stress on the photosystem II in plants (Murchie and Lawson 2013). In present research, $P$. indica increased $\mathrm{Fv} / \mathrm{Fm}$ both in control and osmotic stressed plants of rice. Previously Sherameti et al. (2008) and Bakshi et al. (2017) reported that $P$. indica increased the level of Fv/ Fm in Arabidopsis thaliana under abiotic stress. Similarly, it also stabilized the grana in chloroplasts. Under osmotic stress it helped maintaining grana in proper stacked arrangement, thus protecting the photosynthetic system. .
The association with $P$. indica also altered the expression of P5CS. P. indica increased the expression of this gene under both control and stressed environments. This gene is involved in the synthesis of proline, an amino acid which is critical for plant growth and development. Proline acts as both an osmotic agent and a radical scavenger (Kishor et al. 2014; Kishor et al. 2015). The supportive role of $P$. indica in increasing the synthesis of this protective compound was also evidenced by our experiment. The accumulation of proline due to osmotic stress was more than double in inoculated plants of WC-297 and Caawa and almost double in inoculated plants of IR-64, compared to non-inoculated plants. An increase in TAC was also associated with the presence of the endophyte. This increase in TAC was significant in all three genotypes. Increase in proline and TAC accumulation due to the inoculation with $P$. indica has also been reported in other crops by Waller et al. (2005) and Prasad et al. (2013). Most of the rice inoculation with $P$. indica had been performed to mitigate the salinity, heavy metal and some biotic stresses. We have tested the association of $P$. indica with rice plant under osmotic stress and it was summarized that this fungus improved plant performance under water deficient environment as it is already reported for inducing tolerance in rice under many other stresses. 


\section{Conclusion}

$P$. indica is known to help maintaining growth and yield in many field crops under drought stress, but the underlying mechanisms are not fully elucidated. In the present study, $P$. indica was found to improve morphological traits, mineral uptake and protect the photosynthetic machinery in rice plants submitted to osmotic stress. Moreover, the inoculation with $P$. indica increased $\mathrm{Fv} /$ Fm, up regulated the expression of P5CS, raised the accumulation of proline and TAC. These results confirmed that the endophyte has a significant role in protecting the rice plant against osmotic stress. Further investigation is needed, however, to validate these effects of the inoculation in rice fields under various drought scenarios and use the inoculation with $P$. indica as an additional agronomical tool to improve rice grain yield under drought-prone conditions.

\section{Additional file}

Additional file 1: Table S1. Forward and reverse primers of the genes under study. (DOCX $14 \mathrm{~kb}$ )

\section{Abbreviations}

Caawa: Moderately drought tolerant genotype; CDS: Coding DNA sequence; Fv/ Fm: Maximum quantum yield of PSII; IR-64: Drought susceptible genotypes; P: Phosphorus; P. indica: Piriformospora indica; P5CS: Pyrroline-5-carboxylate synthase; PEG-6000: Polyethylene glycol-6000; ROS: Reactive oxygen species; TAC: Total antioxidant capacity; TEM: Transmission electron microscope; WC297: Drought tolerant genotypes; Zn: Zinc

\section{Acknowledgments \\ Research in the author's laboratories was financed by the Higher Education Commission of Pakistan, 973 National Project (517102-N51501/001), National Natural Science Foundation of China Project, International (Regional) Cooperation and Exchange Program (517102-N11808ZJ), The Fundamental Research Funds for the Central Universities (517102*172210172) and Jiangsu Collaborative Innovation Center for Modern Crop Production (JCIC-MCP). P. indica was provided by Dr. Alga Zuccaro, Max Planck Institute for Terrestrial Microbiology, Germany and Dr. Bing-Gan Lou, Institute of Biotechnology, Zhejiang University, Hangzhou, People's Republic of China.}

\section{Funding}

This work was funded by the Higher Education Commission of Pakistan. Project: Indigenous 5000 PhD Fellowship, Pin No.: 213-66438-2Av2-077, International Research Support Initiative Program (IRSIP), HEC project \# 2297 and 973 national project (517102-N51501/001), National Natural Science Foundation of China Project, International (Regional) Cooperation and Exchange Program (517102-N11808ZJ), the Fundamental Research Funds for the Central Universities (517102*172210172) and Jiangsu Collaborative Innovation Center for Modern Crop Production (JCIC-MCP).

\section{Availability of data and materials}

All relevant data are provided as figures within the paper. Excel files can be provided on demand.

\section{Authors' contributions}

Conceived and designed the experiments: MABS ZA ASK. Performed the experiments: MABS. Analyzed the data: MABS ZA IHS. Contributed reagents/ materials/analysis tools: ZA IHS IAR. Wrote the paper: MABS. Critical review and editing: ZA ASK IAR. All authors read and approved the final manuscript.

Ethics approval and consent to participate

There has no ethics problems involved in this article.

\section{Consent for publication}

These co-authors involved in the paper all consent to publish this article on Rice.

\section{Competing interests}

The authors declare that they have no competing interests.

\section{Publisher's Note}

Springer Nature remains neutral with regard to jurisdictional claims in published maps and institutional affiliations.

\section{Author details}

${ }^{1}$ Department of Plant Breeding and Genetics, University of Agriculture, 38040, Faisalabad, Pakistan. ${ }^{2}$ Centre of Agricultural Biochemistry and Biotechnology, University of Agriculture, 38040, Faisalabad, Pakistan. ${ }^{3}$ Department of Agronomy, College of Agriculture and Biotechnology, Zhejiang University, 310058, Hangzhou, People's Republic of China. ${ }^{4}$ Department of Plant Breeding and Genetics, Muhammad Nawaz Shareef University of Agriculture, 60000, Multan, Pakistan.

Received: 5 January 2018 Accepted: 30 April 2018

Published online: 24 May 2018

\section{References}

Abadi VAJM, Sepehri M (2016) Effect of Piriformospora indica and Azotobacter chroococcum on mitigation of zinc deficiency stress in wheat (Triticum aestivum L.). Symbiosis 69(1):9-19

Abo-Doma A, Edrees S, Abdel-Aziz S (2016) The effect of mycorrhiza growth and expression of some genes in barley. Egypt J Genet Cytol 40(2):301-313

Ashraf M, Foolad M (2007) Roles of glycine betaine and proline in improving plant abiotic stress resistance. Environ Exp Bot 59(2):206-216

Bagheri A, Saadatmand S, Niknam V, Nejadsatari T, Babaeizad V (2014) Effects of Piriformospora indica on biochemical parameters of Oryza sativa under salt stress. Int J Biosci 4(4):24-32

Bagheri AA, Saadatmand S, Niknam V, Nejadsatari T, Babaeizad V (2013) Effect of endophytic fungus, Piriformospora indica, on growth and activity of antioxidant enzymes of rice (Oryza sativa L.) under salinity stress. Int J Adv Biol Biomed Res 1(11):1337-1350

Bakshi M, Sherameti I, Meichsner D, Thürich J, Varma A, Johri AK, Yeh K-W, Oelmüller $R$ (2017) Piriformospora indica reprograms gene expression in Arabidopsis phosphate metabolism mutants but does not compensate for phosphate limitation. Front Microbiol 8:1262

Bates L, Waldren R, Teare I (1973) Rapid determination of free proline for waterstress studies. Plant Soil 39(1):205-207

Cakmak I (2000) Tansley review no. 111 possible roles of zinc in protecting plant cells from damage by reactive oxygen species. New Phytol 146(2):185-205

Ding L, Li Y, Li Y, Shen Q, Guo S (2014) Effects of drought stress on photosynthesis and water status of rice leaves. Chin J Rice Sci 28(1):65-70

Fahad S, Bajwa AA, Nazir U, Anjum SA, Faroog A, Zohaib A, Sadia S, Nasim W, Adkins S, Saud S (2017) Crop production under drought and heat stress: plant responses and management options. Front Plant Sci 8:1147

Fahramand M, Mahmoody M, Keykha A, Noori M, Rigi K (2014) Influence of abiotic stress on proline, photosynthetic enzymes and growth. Int Res J Appl Basic Sci 8(3):257-265

Farooq M, Wahid A, Kobayashi N, Fujita D, Basra S (2009a) Plant drought stress: effects, mechanisms and management. Agron Sustain Dev 29(1):185-212

Farooq M, Wahid A, Lee D-J, Ito O, Siddique KH (2009b) Advances in drought resistance of rice. Crit Rev Plant Sci 28(4):199-217

Food and Agriculture Organization of the United Nations. http://www.fao.org/3/ I8317EN/l8317EN.pdf. Accessed 25 Dec 2017.

Franken P (2012) The plant strengthening root endophyte Piriformospora indica: potential application and the biology behind. Appl Microbiol Biotechnol 96(6):1455-1464

Gill SS, Gill R, Trivedi DK, Anjum NA, Sharma KK, Ansari MW, Ansari AA, Johri AK, Prasad R, Pereira E (2016) Piriformospora indica: potential and significance in plant stress tolerance. Front Microbiol 7:1-20

Gill SS, Tuteja N (2010) Reactive oxygen species and antioxidant machinery in abiotic stress tolerance in crop plants. Plant Physiol Biochem 48(12):909-930

Haefele SM, Kato Y, Singh S (2016) Climate ready rice: augmenting drought tolerance with best management practices. Field Crop Res 190:60-69 
Hattori T, Inanaga S, Araki H, An P, Morita S, Luxova M, Lux A (2005) Application of silicon enhanced drought tolerance in Sorghum bicolor. Physiol Plant 123(4):459-466

Hill TW, Kafer E (2001) Improved protocols for aspergillus minimal medium: trace element and minimal medium salt stock solutions. Fungal Genet Rep 48(1):20-21

Im YJ, Han O, Chung GC, Cho BH (2002) Antisense expression of an Arabidopsis omega-3 fatty acid desaturase gene reduces salt/drought tolerance in transgenic tobacco plants. Mol Cells 13(2):264-271

Ji K, Wang Y, Sun W, Lou Q, Mei H, Shen S, Chen H (2012) Drought-responsive mechanisms in rice genotypes with contrasting drought tolerance during reproductive stage. J Plant Physiol 169(4):336-344

Jogawat A, Saha S, Bakshi M, Dayaman V, Kumar M, Dua M, Varma A, Oelmüller R, Tuteja N, Johri AK (2013) Piriformospora indica rescues growth diminution of rice seedlings during high salt stress. Plant Signal Behav 8(10):e26891

Kaya MD, Okçu G, Atak M, Çıkılı Y, Kolsarıcı Ö (2006) Seed treatments to overcome salt and drought stress during germination in sunflower (Helianthus annuus L.). Eur J Agron 24(4):291-295

Kishor K, Polavarapu B, Sreenivasulu N (2014) Is proline accumulation per se correlated with stress tolerance or is proline homeostasis a more critical issue? Plant Cell Environ 37(2):300-311

Kishor PBK, Kumari PH, Sunita M, Sreenivasulu N (2015) Role of proline in cell wall synthesis and plant development and its implications in plant ontogeny. Front Plant Sci 6:1-17

Korres N, Norsworthy J, Burgos N, Oosterhuis D (2017) Temperature and drought impacts on rice production: an agronomic perspective regarding short-and long-term adaptation measures. Water Resour Rural Dev 9:12-27

Li X, Zhang L, Li Y (2011) Preconditioning alters antioxidative enzyme responses in rice seedlings to water stress. Procedia Environ Sci 11:1346-1351

Liu R, Zhang H, Chen Z, Shahid M, Fu X, Liu X (2015) Drought-tolerant rice germplasm developed from an Oryza officinalis transformation-competent artificial chromosome clone. Genet Mol Res 14(4):13667-13678

Michal Johnson J, Sherameti I, Ludwig A, Nongbri PL, Sun C, Lou B, Varma A, Oelmüller R (2011) Protocols for Arabidopsis thaliana and Piriformospora indica co-cultivation-a model system to study plant beneficial traits. Endocytobiosis Cell Res 21:101-113

Michel BE, Kaufmann MR (1973) The osmotic potential of polyethylene glycol 6000. Plant Physiol 51(5):914-916

Mohd S, Shukla J, Kushwaha AS, Mandrah K, Shankar J, Arjaria N, Saxena PN, Narayan R, Roy SK, Kumar M (2017) Endophytic Fungi Piriformospora indica mediated protection of host from arsenic toxicity. Front Microbiol 8:754

Murchie EH, Lawson T (2013) Chlorophyll fluorescence analysis: a guide to good practice and understanding some new applications. J Exp Bot 64(13):3983-3998

Nakashima K, Yamaguchi-Shinozaki K, Shinozaki K (2014) The transcriptional regulatory network in the drought response and its crosstalk in abiotic stress responses including drought, cold, and heat. Front Plant Sci 5:1-7

Nassimi Z, Taheri P (2017) Endophytic fungus Piriformospora indica induced systemic resistance against rice sheath blight via affecting hydrogen peroxide and antioxidants. Biocontrol Sci Technol 27(2):252-267

Ngwene B, Boukail S, Söllner L, Franken P, Andrade-Linares D (2016) Phosphate utilization by the fungal root endophyte Piriformospora indica. Plant Soil 405(1-2):231-241

Padash A, Shahabivand S, Behtash F, Aghaee A (2016) A practicable method for zinc enrichment in lettuce leaves by the endophyte fungus Piriformospora indica under increasing zinc supply. Sci Hortic 213:367-372

Prasad KN, Yang B, Yang S, Chen Y, Zhao M, Ashraf M, Jiang Y (2009) Identification of phenolic compounds and appraisal of antioxidant and antityrosinase activities from litchi (Litchi sinensis Sonn.) seeds. Food Chem 116(1):1-7

Prasad R, Kamal S, Sharma PK, Oelmüller R, Varma A (2013) Root endophyte Piriformospora indica DSM 11827 alters plant morphology, enhances biomass and antioxidant activity of medicinal plant Bacopa monniera. J Basic Microbiol 53(12):1016-1024

Prieto P, Pineda M, Aguilar M (1999) Spectrophotometric quantitation of antioxidant capacity through the formation of a phosphomolybdenum complex: specific application to the determination of vitamin E. Anal Biochem 269(2):337-341

Sarma M, Kumar V, Saharan K, Srivastava R, Sharma A, Prakash A, Sahai V, Bisaria V (2011) Application of inorganic carrier-based formulations of fluorescent pseudomonads and Piriformospora indica on tomato plants and evaluation of their efficacy. J Appl Microbiol 111(2):456-466
Serraj R, McNally KL, Slamet-Loedin I, Kohli A, Haefele SM, Atlin G, Kumar A (2011) Drought resistance improvement in rice: an integrated genetic and resource management strategy. Plant Prod Sci 14(1):1-14

Shahabivand S, Parvaneh A, Aliloo AA (2017) Root endophytic fungus Piriformospora indica affected growth, cadmium partitioning and chlorophyll fluorescence of sunflower under cadmium toxicity. Ecotoxicol Environ Saf 145:496-502

Sherameti I, Tripathi S, Varma A, Oelmüller R (2008) The root-colonizing endophyte Pirifomospora indica confers drought tolerance in Arabidopsis by stimulating the expression of drought stress-related genes in leaves. Mol Plant-Microbe Interact 21(6):799-807

Sokoto M, Muhammad A (2014) Response of rice varieties to water stress in Sokoto, Sudan savannah, Nigeria. J Biosci Med 2(01):68

Swapna S, Shylaraj KS (2017) Screening for osmotic stress responses in Rice varieties under drought condition. Rice Sci 24(5):253-263

Taiz L, Zeiger E (2006) Plant physiology. 4th. Sinauer associate, Sunderland, mass, EUA

Tariq A, Pan K, Olatunji OA, Graciano C, Li Z, Sun F, Sun X, Song D, Chen W, Zhang A (2017) Phosphorous application improves drought tolerance of Phoebe zhennan. Front Plant Sci 8:1561

Uga Y, Kitomi Y, Yamamoto E, Kanno N, Kawai S, Mizubayashi T, Fukuoka S (2015) A QTL for root growth angle on rice chromosome 7 is involved in the genetic pathway of DEEPER ROOTING 1. Rice 8(1):8

Vahabi K, Dorcheh SK, Monajembashi S, Westermann M, Reichelt M, Falkenberg D, Hemmerich P, Sherameti I, Oelmüller R (2016) Stress promotes Arabidopsis-Piriformospora indica interaction. Plant Signal Behav 11(5): e1136763

Varma A, Chordia P, Bakshi M, Oelmüller R (2013) Introduction to Sebacinales. In: Piriformospora indica: Springer; pp 33:3-24

Varma A, Singh A, Sahay NS, Sharma J, Roy A, Kumari M, Rana D, Thakran S, Deka D, Bharti K (2001) Piriformospora indica: an axenically culturable mycorrhizalike endosymbiotic fungus. In: Fungal associations: Springer; pp 9:125-150

Verslues PE, Sharma S (2010) Proline metabolism and its implications for plantenvironment interaction. The arabidopsis book:e0140. https://doi.org/10. 1199/tab.0140

Waller F, Achatz B, Baltruschat H, Fodor J, Becker K, Fischer M, Heier T, Hückelhoven R, Neumann C, von Wettstein D (2005) The endophytic fungus Piriformospora indica reprograms barley to salt-stress tolerance, disease resistance, and higher yield. Proc Natl Acad Sci U S A 102(38):13386-13391

Yang P-M, Huang Q-C, Qin G-Y, Zhao S-P, Zhou J-G (2014) Different droughtstress responses in photosynthesis and reactive oxygen metabolism between autotetraploid and diploid rice. Photosynthetica 52(2):193-202

Yoshida, S (1976) Routine procedure for growing rice plants in culture solution. Laboratory manual for physiological studies of rice. 61-66

Yue B, Xue W, Xiong L, Yu X, Luo L, Cui K, Jin D, Xing Y, Zhang Q (2006) Genetic basis of drought resistance at reproductive stage in rice: separation of drought tolerance from drought avoidance. Genetics 172(2):1213-1228

Zain NAM, Ismail MR, Mahmood M, Puteh A, Ibrahim MH (2014) Alleviation of water stress effects on MR220 rice by application of periodical water stress and potassium fertilization. Molecules 19(2):1795-1819

\section{Submit your manuscript to a SpringerOpen ${ }^{\mathcal{O}}$ journal and benefit from:}

- Convenient online submission

- Rigorous peer review

- Open access: articles freely available online

- High visibility within the field

- Retaining the copyright to your article

Submit your next manuscript at $>$ springeropen.com 